\title{
Síntese e Caracterização de Poliuretanos Aniônicos em Dispersão Aquosa à Base de Polibutadieno Líquido Hidroxilado, Poli(glicol propilênico) e Diisocianato de Isoforona
}

\author{
Fernanda M. B. Coutinho \\ Departamento de Processos Industriais, IQ/UERJ e UFRJ/IMA \\ Marcia C. Delpech e Lairton S. Alves \\ Instituto de Macromoléculas Professora Eloisa Mano, IMA/UFRJ
}

\begin{abstract}
Resumo: Foram sintetizados sob a forma de dispersões aquosas estáveis, com viscosidade aparente na faixa de 30 a $220 \mathrm{cP}$, poliuretanos à base de polibutadieno líquido hidroxilado (HTPB) de massa molecular numérica média 3500, poli(glicol propilênico) (PPG) de massa molecular viscosimétrica média 2490, diisocianato de isoforona (IPDI) e ácido dimetilolpropiônico (DMPA). O tamanho médio de partícula das dispersões foi determinado e as propriedades mecânicas dos filmes vazados avaliadas. As dispersões foram aplicadas em madeira formando revestimentos homogêneos cuja adesividade foi determinada. A inserção de HTPB na cadeia poliuretânica provocou um aumento no tamanho de partícula das dispersões aquosas, proporcionou uma maior resistência mecânica aos filmes e aumentou a adesividade dos revestimentos ao substrato.
\end{abstract}

Palavras-chave: $H T P B, P P G, I P D I, D M P A$, poliuretanos, dispersões aquosas.

Synthesis and characterization of waterborne anionic polyurethanes based on hydroxy terminated polybutadiene, poly(propylene glycol) and isophorone diisocyanate

Abstract: Non-polluting systems based on waterborne polyurethanes were obtained from hydroxy-terminated polybutadiene (HTPB) ('Mn = 3500), poly(propylene glycol) ( $\mathrm{PPG}$ (' $\mathrm{Mv}=2490)$, isophorone diisocyanate (IPDI) and dimethylolpropionic acid (DMPA). The aqueous dispersions, with $35 \%$ of solid content, were stable for nearly a month and their apparent viscosity ranged from 30 to $220 \mathrm{cP}$. An increase in HTPB content in the formulations led to an increase in the average particle size of the dispersions. This increase also promoted an improvement in the mechanical resistance of cast films from the aqueous dispersions. The adhesive properties of dispersions as wood coatings were determined and it was observed that the presence of HTPB improved the adhesion between coating and substrate.

Keywords: $H T P B, P P G, I P D I, D M P A$, polyurethanes, aqueous dispersions

\section{Introdução}

Revestimentos obtidos a partir de dispersões aquosas têm tido uma intensa participação no mercado nos últimos anos ${ }^{[1]}$. A crescente preocupação com questões ambientais deixou patente a necessidade da substituição de solventes orgânicos, cujas emissões para a atmosfera são tóxicas e causam danos ao meio ambiente, por água na obtenção e/ou aplicação de revestimentos ${ }^{[2-4]}$.

A crescente aplicação das dispersões aquosas de poliuretanos como revestimentos está baseada em

Autor para correspondência: Fernanda M. B. Coutinho, Departamento de Processos Industriais, IQ/UERJ, Rio de Janeiro, RJ e UFRJ/IMA, C.P. 68525, CEP: 21945-970, Rio de Janeiro, RJ, E-mail: fern@ima.ufr.br. 
uma grande variedade de propriedades que incluem a capacidade de formar filmes à temperatura ambiente, resistência química a solventes, resistência à abrasão e flexibilidade ${ }^{[2-5]}$. Essas dispersões apresentam ainda ótimas características de adesão a diversos tipos de substratos como vidro, tecidos, metais, papel, porcelana, cimento, couro, madeira, concreto e a superfícies poliméricas $^{[6-8]}$.

Os poliuretanos, assim como a maioria dos polímeros sintéticos, não são solúveis em meio aquoso e, por isso, é necessário que sejam feitas modificações na cadeia para torná-los suscetíveis à dispersão em água ${ }^{[2,6-9]}$. Grupos iônicos ou segmentos hidrofílicos, que podem estar presentes nos monômeros ou no extensor de cadeia, permitem que o polímero se torne compatível com o meio aquoso ${ }^{[10]}$.

Uma grande variedade de polióis podem ser empregados na síntese de poliuretanos em dispersão aquosa. Polióis poliéteres e poliésteres bem como misturas de ambos são os mais utilizados industrialmente. No entanto, outros polímeros contendo grupos hidroxila terminais como politioéteres, poliaminoéteres, poliacetais, policarbonatos, polímeros naturais, polissiloxanos e polibutadieno líquido também podem ser empregados ${ }^{[6,7,11]}$.

A utilização do polibutadieno líquido hidroxilado (HTPB) na síntese de poliuretanos em meio orgânico tem crescido muito nos últimos anos ${ }^{[7]}$. O uso desse poliol é vantajoso devido à presença da cadeia hidrocarbônica apolar que aumenta a estabilidade dos poliuretanos frente à hidrólise. Outra vantagem é em termos de processamento e a possibilidade da presença de grande quantidade de carga na formulação, o que leva a um barateamento do produto ${ }^{[12,13]}$.

Poliuretanos derivados do HTPB são empregados como aglutinantes na produção de propelentes sólidos para foguetes ${ }^{[13,14]}$ e são responsáveis pela estabilidade dimensional do comburente ${ }^{[7,15]}$. Também são empregados na produção de adesivos com elevadas resistências química e à umidade e alta flexibilidade ${ }^{[13]}$. Outra utilização importante desses materiais é no preparo de membranas para separação de gases devido a sua flexibilidade a baixas temperaturas e alta segregação entre os segmentos rígidos e flexíveis ${ }^{[16,17]}$.

São raras as publicações sobre a síntese de poliuretanos à base de HTPB dispersos em água ${ }^{[18]}$. O desenvolvimento desses materiais é de grande importância para vários segmentos da indústria tornando
Tabela 1. Quantidades (em massa) dos reagentes empregados na formulação correspondente à razão $\mathrm{NCO} / \mathrm{OH}=1,5$ e $50 \%$ de HTPB em relação à massa de pré-polímero

\begin{tabular}{cc}
\hline Reagentes & Massa (g) \\
\hline HTPB & 45,5 \\
PPG & 20,0 \\
DMPA & 4,6 \\
IPDI & 20,9 \\
DBTDL & 0,01 \\
Pré-polímero & 91,0 \\
TEA & 3,5 \\
H 20 & 179,2 \\
EDA & 2,0 \\
\hline
\end{tabular}

Observações

- Percentual de HTPB = 50 \% em relação à massa de pré-polímero

- Percentual de DMPA $=5 \%$ em relação à massa de pré-polímero

- Cálculo da quantidade de EDA foi feito em função do teor de grupamentos NCO terminais

- A quantidade de água adicionada foi suficiente para a obtenção de dispersão com 35 \% de sólidos totais.

possível a obtenção de resinas versáteis, não-poluentes e de fácil aplicação como revestimentos devido à baixa viscosidade do meio ${ }^{[6,7]}$.

No Instituto de Macromoléculas Professora Eloisa Mano da UFRJ ${ }^{[6,7]}$, foi desenvolvido um método de síntese de poliuretanos em dispersão aquosa à base de HTPB, poli(glicol propilênico) (PPG), diisocianato de isoforona (IPDI) e ácido dimetilolpropiônico (DMPA) que levou à formação de dispersões estáveis. Foram avaliadas as propriedades mecânicas dos filmes obtidos por vazamento e adesivas dos revestimentos aplicados em madeira. $\mathrm{O}$ objetivo deste estudo é o desenvolvimento de dispersões aquosas estáveis para aplicação, principalmente, como revestimentos para madeira e papel.

\section{Experimental}

\section{Materiais}

Os principais reagentes utilizados na síntese dos poliuretanos foram usados como recebidos e estão 
listados a seguir. Ácido dimetilolpropiônico (DMPA), da Aldrich Chemical Company, Inc.; dibutildilaurato de estanho (DBTDL), da Aldrich Chemical Company, Inc.; diisocianato de isoforona (3-isocianatometil3,5,5-trimetil-hexil-isocianato) (IPDI), concentração: 98,6\% ${ }^{[19]}$, da Hülls AG, doado pela Petroflex; etilenodiamina (EDA) (solução a 98,8\%), grau de pureza PA-ACS, da Reagen Quimibrás Indústrias
Químicas SA; polibutadieno líquido hidroxilado (HTPB), Petroflex, massa molecular numérica média de 3500, determinada por osmometria de pressão de vapor; poli(glicol propilênico) (PPG) (Voranol 2120) doado pela Dow Química SA, número de hidroxilas $49,81^{[20]}$ e massa molecular viscosimétrica média de 2490 , seco sob vácuo a $120^{\circ} \mathrm{C}$, por 3 horas ${ }^{[2,6,7]}$; e trietilamina (TEA), da Union Carbide.

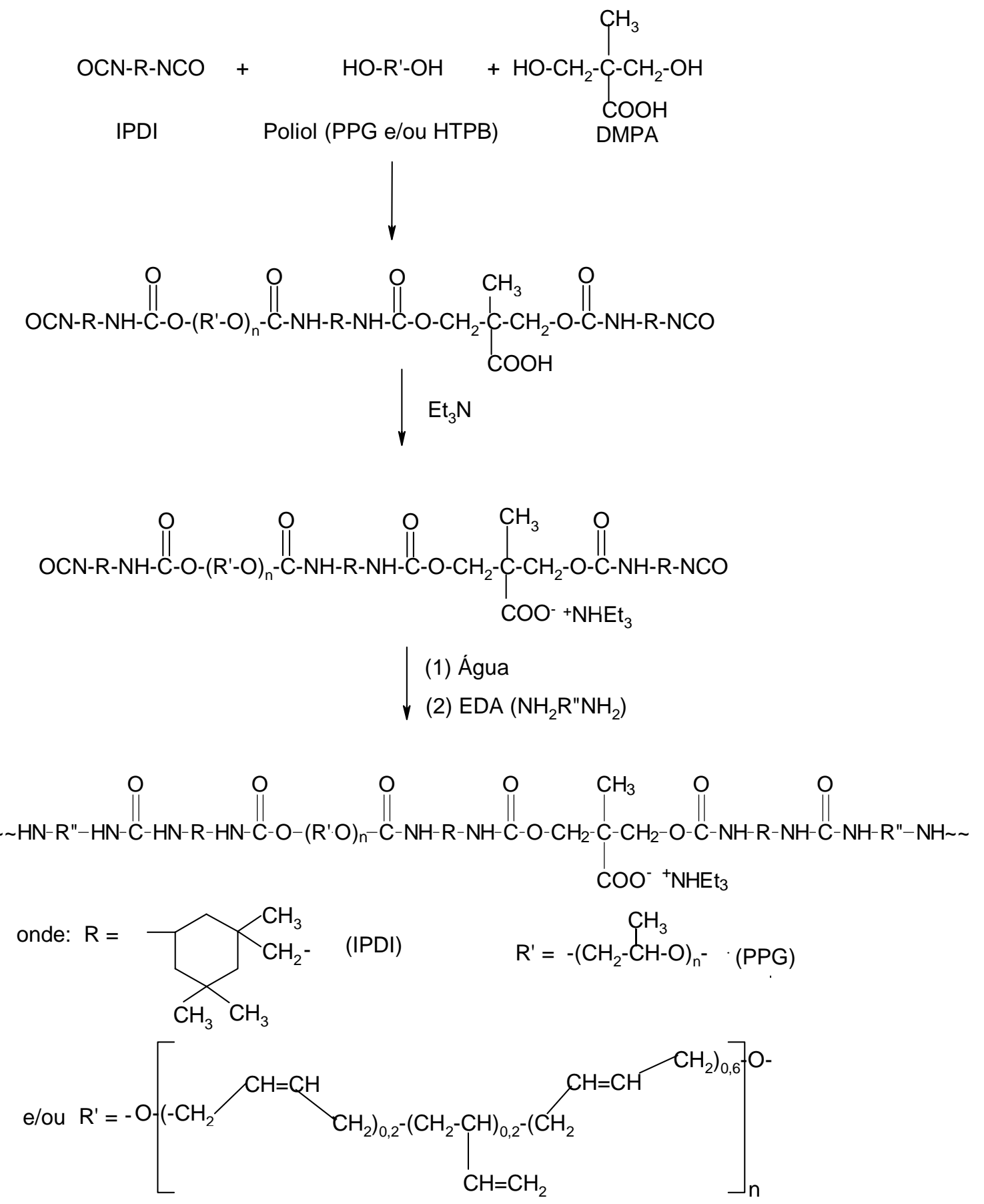

Figura 1. Síntese dos poliuretanos dispersos em água 


\section{Síntese das dispersões aquosas}

A síntese do pré-polímero à base de HTPB, PPG, DMPA e IPDI foi catalisada por dibutildilaurato de estanho (DBTDL), em ausência de solvente. A reação foi conduzida a $100^{\circ} \mathrm{C}$, em atmosfera inerte, por 2 horas. Em seguida, a temperatura foi reduzida para $50^{\circ} \mathrm{Ce}$ foram retiradas alíquotas da mistura reacional para a dosagem do teor de grupamentos isocianato $(\mathrm{NCO})$ terminais do pré-polímero ${ }^{[19]}$.

A neutralização dos grupos carboxila, provenientes do DMPA, com TEA foi conduzida a $50^{\circ} \mathrm{C}$, durante 1 hora. $\mathrm{O}$ ionômero formado foi então disperso em água, a $25^{\circ} \mathrm{C}$ e foi imediatamente adicionado o extensor de cadeia aminado (EDA), em quantidade estequiométrica, calculada de acordo com o valor obtido na dosagem dos grupamentos NCO terminais do pré-polímero. A reação de extensão de cadeia, no meio aquoso, foi então conduzida a $35^{\circ} \mathrm{C}$. A Figura 1 mostra, de forma esquemática, a síntese das resinas uretânicas. A Tabela 1 apresenta as quantidades de reagentes empregadas em uma das formulações.

Nas dispersões poliuretânicas, variou-se de $0 \mathrm{a}$ $50 \%$ o percentual de HTPB em relação à massa de pré-polímero. Foram sintetizados pré-polímeros sempre com 5\% de DMPA em relação à massa de prépolímero e foram utilizadas duas razões entre o número de equivalentes-grama de diisocianato e de dihidroxilados $(\mathrm{NCO} / \mathrm{OH}), 1,5$ e 3,0. Foi observado experimentalmente que não era possível dispersar prépolímeros com razões $\mathrm{NCO} / \mathrm{OH}$ inferiores a 1,5, devido à alta viscosidade do meio reacional. Dispersões com razões $\mathrm{NCO} / \mathrm{OH}$ superiores a 3,0 produziram filmes quebradiços ${ }^{[6]}$.

Caracterização das dispersões aquosas, dos filmes vazados e dos revestimentos aplicados em madeira

O tamanho médio de partícula das dispersões foi determinado em analisador de tamanhos de partícula por espalhamento de luz laser Malvern Sizer Microplus MAF 5001. O comprimento de onda da luz laser foi de $633 \mathrm{~nm}$. As dispersões foram diluídas em água destilada e deionizada, filtradas em tela de nylon e submetidas a ultra-som, dentro do próprio equipamento, de modo que possíveis aglomerados, porventura formados, fossem desagregados.

As dispersões aquosas foram vazadas em superfícies niveladas revestidas com teflon. Foram obtidos filmes após secagem por 1 semana à temperatura ambiente e por 12 horas em estufa, a $60^{\circ} \mathrm{C}^{[2,6,7,21]}$. A espessura dos filmes obtidos variou de 0,3 a $0,8 \mathrm{~mm}$.

Os ensaios de tração dos filmes vazados foram feitos em Máquina Universal de Ensaios Instron 4202, com garras pneumáticas A2-170 e célula de $1 \mathrm{KN}$. Os corpos de prova eram retangulares $(5 \times 70) \mathrm{mm}^{2} \mathrm{e}$ a velocidade de separação das garras foi de $500 \mathrm{~mm} /$ min, de acordo com método adaptado da literatura ${ }^{[2,6]}$.

Testes de adesividade dos revestimentos, formados pelas dispersões aquosas após aplicação em madeira, foram executados segundo método padrão ${ }^{[22,23]}$ adaptado $^{[3,4,6,7]}$ com dinamômetro portátil Elcometer 106 no 2 (faixa de 1 a $7 \mathrm{MPa}$ ), que oferece a vantagem de permitir que o teste seja feito diretamente sobre a superfície revestida (Figura 2). Esse procedimento avalia a adesão de um revestimento a um substrato, pela determinação da tensão mínima, aplicada perpendicularmente, que uma determinada área da superfície do corpo de prova pode suportar até o rompimento, que ocorre no plano ou interface mais fraca do sistema. É um processo simples e comparativo onde o dinamômetro é acoplado a carretéis de alumínio previamente colados com adesivo padrão à superfície revestida. O rompimento pode ocorrer entre o adesivo e o revestimento, entre o revestimento e o substrato ou ainda provocado por falha de coesão no substrato. Nesse último caso, o sistema adesivo/ revestimento/ superfície do substrato permanece intacto.

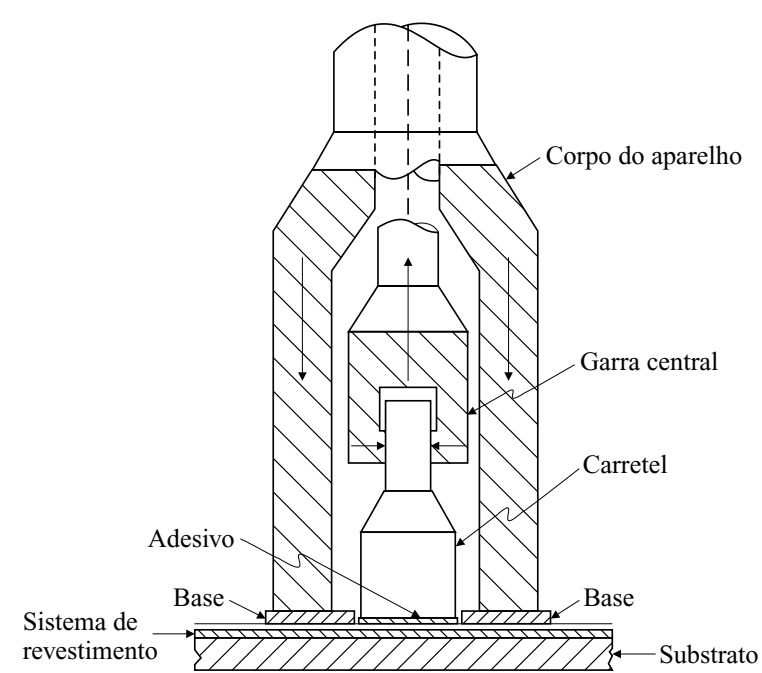

Figura 2. Representação esquemática do dinamômetro portátil ${ }^{[22]}$ 
As dispersões foram aplicadas com pincel em placas de ipê $(20 \mathrm{~cm} \times 20 \mathrm{~cm} \times 1 \mathrm{~cm})$ que secaram à temperatura ambiente por 7 dias. Após esse período, os carretéis foram colados ao revestimento e deixou-se secar por mais 7 dias $^{[6,7]}$.

\section{Resultados e Discussão}

\section{Caracterização das dispersões aquosas}

Foram obtidas dispersões com teor de sólidos de $35 \pm 2,5 \%{ }^{[24]}$ e $\mathrm{pH}$ na faixa de 8 a 10 . A viscosidade aparente das dispersões aquosas foi determinada em viscosímetro Brookfield LVT, a 25,0 \pm $0,1^{\circ} \mathrm{C}$, na taxa de cisalhamento de $30 \mathrm{rpm}$. Os valores obtidos ficaram dentro da faixa de 30 a $220 \mathrm{cP}$, sendo que as dispersões contendo teores mais altos de HTPB ficaram nos limites superiores. A estabilidade das dispersões foi observada em termos de sedimentação do sólido. As dispersões foram consideradas estáveis (ausência de sedimentação), levando-se em conta o período de até 1 mês após a síntese desse material.

A Tabela 2 mostra os valores de tamanhos de partícula obtidos para as dispersões aquosas.

Uma partícula de poliuretano típica dispersa é formada por uma camada externa composta de porções hidrofílicas da molécula que podem ser constituídas de segmentos hidrofílicos, contendo ou não grupos iônicos, e também de ligações uretânicas e urêicas ${ }^{[6,25]}$. O teor dessa porção hidrofílica tem influência direta sobre o tamanho da partícula ${ }^{[26]}$. Nas partículas provenientes das dispersões analisadas, a camada externa deve ser formada pelos grupos carboxilato provenientes do DMPA, ligações uretânicas provenientes do prépolímero e urêicas, resultantes da reação de extensão de cadeia. A camada interna, hidrofóbica, é formada predominantemente pelos segmentos hidrofóbicos do PPG e do HTPB.

Os resultados apresentados na Tabela 2 mostram que a inserção de HTPB na cadeia provocou um aumento nos tamanhos de partícula e que a sua influência foi pouco significativa para mais baixos percentuais (5 e 10\%). Pode-se observar ainda que os valores de tamanho de partícula se elevaram drasticamente quando se empregou $50 \%$ de HTPB. Esse aumento que pode ser devido à estrutura não-linear e irregular do HTPB, devido à presença de unidades repetitivas de diferentes configurações (1,4-trans, 1,4-cis e 1,2-vi-
Tabela 2. Tamanho médio de partícula das dispersões aquosas

\begin{tabular}{ccc}
\hline \% HTPB & $\mathbf{N C O} / \mathbf{O H}=\mathbf{1 , 5}(\boldsymbol{\mu m})$ & $\mathbf{N C O} / \mathbf{O H}=\mathbf{3 , 0}(\boldsymbol{\mu m})$ \\
\hline 0 & 0,65 & 0,22 \\
5 & 3,80 & 0,55 \\
10 & 3,52 & 0,55 \\
30 & 4,02 & 2,35 \\
50 & 82,46 & 12,57 \\
\hline
\end{tabular}

Os percentuais de HTPB e DMPA (fixo em 5 \%) estão expressos em relação à massa de pré-polímero.

nil), dificultando assim a compactação das cadeias uretânicas na formação da partícula ${ }^{[6]}$. O alto caráter hidrofóbico do HTPB também pode dificultar a dispersão, levando à formação de partículas maiores.

Comparando-se as razões $\mathrm{NCO} / \mathrm{OH} 1,5$ e 3,0, verifica-se que a primeira, que apresenta mais baixo teor de segmentos rígidos, ou seja, um menor número de ligações uretânicas e urêicas, levou à formação de partículas maiores, pois o poliuretano resultante tem menor teor de sítios hidrofílicos em suas cadeias. A razão $\mathrm{NCO} / \mathrm{OH}$ mais alta $(3,0)$ favoreceu a formação de partículas menores. No entanto, essas cadeias apresentam mais alto teor de segmentos rígidos, o que poderia dificultar a formação de partículas pequenas. Pode-se supor então, que a presença de uma maior proporção de ligações uretânicas e urêicas (sítios hidrofílicos) exerceu uma influência mais forte sobre o tamanho de partícula do que a rigidez da cadeia.

\section{Avaliação mecânica dos filmes obtidos por vazamento das dispersões aquosas}

Nos filmes das dispersões contendo HTPB incorporado na cadeia poliuretânica, observou-se um ligeiro amarelamento, que não ocorreu nas dispersões sem a presença desse poliol. Isso é devido à característica de o HTPB sofrer processos termooxidativos devido à presença de hidrogênios alílicos em sua cadeia ${ }^{[7]}$.

O filme obtido a partir da dispersão preparada com razão $\mathrm{NCO} / \mathrm{OH}=1,5$ e sem $\mathrm{HTPB}$ apresentou superfície muito pegajosa (com muito tack). Normalmente, o tack é verificado pelo toque da superfície com o dedo ${ }^{[27]}$. À medida que a proporção de HTPB incorporada à cadeia poliuretânica aumentou, o tack foi diminuindo gradualmente até desaparecer, quando o teor de HTPB atingiu 50\%. Os filmes obtidos eram flexíveis e transparentes. Para a razão $\mathrm{NCO} / \mathrm{OH}=3,0$, o filme prove- 
Tabela 3. Valores encontrados nos ensaios de tração para os filmes vazados.

\begin{tabular}{cccc}
\hline $\begin{array}{c}\text { Reação } \\
\mathrm{NCO} / \mathrm{OH} / \% \text { HTPB }\end{array}$ & $\varepsilon(\%)$ & Propriedades Mecânicas \\
\hline 1,5 / 0 & 1461,0 & $\mathrm{E}(\mathrm{MPa})$ & $\sigma(\mathrm{MPa})$ \\
1,5 / 15 & 978,6 & 0,74 & 0,40 \\
1,5 / 20 & 902,4 & 1,39 & 7,42 \\
1,5 / 30 & 788,1 & 2,41 & 9,16 \\
$1,5 / 50$ & 509,1 & 3,18 & 9,97 \\
$3,0 / 0$ & 493,0 & 10,25 & 10,07 \\
$3,0 / 10$ & 471,1 & 67,69 & 22,60 \\
\hline
\end{tabular}

$\varepsilon$ - Alongamento na ruptura; E - Módulo de elasticidade; $\sigma$ - Tensão na ruptura

niente da dispersão sem HTPB não apresentou tack e se mostrou mais rígido. Essa rigidez foi aumentando com a incorporação de HTPB até 30\%, quando o filme se tornou enrugado e quebradiço.

A Tabela 3 mostra os resultados dos ensaios mecânicos obtidos para os filmes vazados.

Pode-se observar que o aumento no teor de HTPB levou ao aumento tanto no módulo de elasticidade quanto na tensão na ruptura e à diminuição no alongamento na ruptura. Isso mostra que o HTPB aumenta a resistência do material, diminuindo sua elasticidade. Essa resistência aumenta, ainda mais, quando o teor de ligações uretânicas e urêicas é mais alto $(\mathrm{NCO} / \mathrm{OH}=3,0)$, devido ao aumento das interações por ligações hidrogênio. No entanto, os filmes obtidos a partir de formulações com $\mathrm{NCO} /$ $\mathrm{OH}=3,0$ e com teor de HTPB $>30 \%$ em massa se apresentaram quebradiços. Isso mostra que há um limite para essa incorporação, já que o HTPB também contribui para enrijecer a cadeia.

\section{Testes de adesividade dos revetimentos das disper- sões aplicadas em madeira}

Nos testes de adesividade realizados, foi sempre observada a falha de coesão no substrato, ou seja, a coesão do sistema adesivo/revestimento/substrato foi maior do que a coesão do próprio substrato, o que é indicação de adesão satisfatória do revestimento ao substrato.

A Tabela 4 apresenta os resultados encontrados nas determinações de tensão na ruptura para os revestimentos obtidos a partir das dispersões aquosas aplicadas em madeira.

Apesar da baixa sensibilidade do teste em termos quantitativos, pode-se verificar que comparativamente há uma tendência a aumento na tensão na ruptura à medida que o teor de HTPB incorporado na cadeia aumenta. Isso mostra que as propriedades adesivas das dispersões podem ser favorecidas pela presença desse polímero em suas formulações.

Os revestimentos formados apresentaram características semelhantes aos filmes vazados em relação ao tack, ou seja, quanto maior foi o teor de HTPB na formulação, menos pegajosa se mostrou a superfície. As superfícies dos revestimentos provenientes das dispersões de razão $\mathrm{NCO} / \mathrm{OH}=1,5$ se apresentaram mais pegajosas do que as de razão $\mathrm{NCO} / \mathrm{OH}=3,0^{[6]}$. O tack é uma propriedade que não está diretamente ligada à capacidade de aderência ao substrato, isto é, um revestimento que apresenta uma superfície mais pegajosa (maior tack) pode aderir menos a um substrato do que outro revestimento, de superfície menos pegajosa ${ }^{[27]}$. No entanto, foi observado nos revestimentos testados que o aumento do tack estava ligado diretamente ao aumento da adesividade.

Tabela 4. Valores de tensão na ruptura nos testes de adesão

\begin{tabular}{cc}
\hline $\mathbf{N C O} / \mathbf{O H}$ / \% HTPB & Tensão na ruptura (MPa) \\
\hline $1,5 / 5$ & 4,5 \\
$1,5 / 10$ & 4,5 \\
$1,5 / 20$ & 5,0 \\
$1,5 / 30$ & 5,0 \\
$1,5 / 50$ & 5,5 \\
$3,0 / 20$ & 4,8 \\
$3,0 / 30$ & 5,8 \\
\hline
\end{tabular}

Polímeros: Ciência e Tecnologia, vol. 10, no 1, p. 49-55, 2000 


\section{Conclusões}

O método de síntese desenvolvido mostrou-se eficiente para a obtenção de poliuretanos à base de HTPB dispersos em água. As dispersões permaneceram estáveis pelo período de 1 mês. $\mathrm{O}$ aumento no teor de HTPB incorporado na cadeia poliuretânica promoveu um aumento no tamanho de partícula, na resistência mecânica dos filmes obtidos por vazamento e na adesividade dos revestimentos formados pela aplicação das dispersões aquosas em madeira. O tack na superfície dos filmes e revestimentos formados diminuiu com o aumento do teor de HTPB adicionado à formulação.

\section{Agradecimentos}

Os autores agradecem ao CNPq, CNPq/ PADCT, PRONEX, CEPEL, Dow Química SA e Petroflex pelo apoio recebido.

\section{Referências Bibliográficas}

1. Hoffman, J. - Chem. Mark. Reporter, 252,18, p.FR12 (1997).

2. Coutinho, F.M.B. \& Delpech, M.C. - Polym. Test., 15, p. 103 (1996).

3. Coutinho, F.M.B., Delpech, M.C. \& Alves, L.S. - J. Appl. Polym. Sci., em impressão.

4. Delpech, M.C. \& Coutinho, F.M.B. - Polym. Test., em impressão.

5. Klein, H. \& Schwab, M. - Polym. Paint Col. J., 184, p. 444 (1994).

6. Delpech, M.C. - "Síntese e Caracterização de Poliuretanos Obtidos como Dispersões Aquosas", Tese de Doutorado, Universidade Federal do Rio de Janeiro, Brasil (1996).

7. Alves, L.S. - "Síntese e Caracterização de Poliuretanos em Dispersão Aquosa à Base de Poli(óxido de propileno) e Polibutadieno Líquido Hidroxilado", Tese de Mestrado, Universidade Federal do Rio de Janeiro, Brasil (1999).

8. Lay, D. \& Cranley, P. - Adhesives Age, p. 6, May (1994).
9. Kim, T.K. \& Kim, B.K. - Colloid Polym. Sci., 269, p. 889 (1991).

10. Loewrigkeit, P. \& VanDyk, K.A. - EP 0189945 A2 (1996).

11. Coogan, R.G. \& Vartan-Boghossian, R. - EP 0404371 A1 (1990).

12. Akbas, A. \& Aksoy, S. - Polymer, 35, p. 2568 (1994).

13. Vilar, W.D. - "Química e tecnologia de poliuretanos", Grupo Pronor, Bahia (1993)

14. Haska, S.B; Bayramli, E.; Pekel, F. \& Özrak, S - J. Appl. Polym. Sci., 64, p. 2347 (1997).

15. Rezende, L.C. - "Estudo Cinético da Reação entre Polibutadienos Hidroxilados e Diisocianatos", Tese de Mestrado, Universidade Federal do Rio de Janeiro (1984).

16. Huang, S.L. \& Lai, J.Y. - Eur. Polym. J., 33, p. 1563 (1997).

17. Huang, S.L. \& Lai, J.Y. - J. Appl. Polym. Sci., 58, p. 1913 (1995).

18. Frisch, K.C.; Xiao, H.X. \& Suthar, B. - EP 807647 (1997)

19. "Book of American Society for Testing and Materials Standards - ASTM" - D 1638-74 (1979).

20. "Book of American Society for Testing and Materials Standards - ASTM" - D 4274-88 (1992).

21. Chen, Y.; Chen, Y.L. - J.Appl. Polym. Sci., 46, p. 435 (1992).

22. "Book of American Society for Testing and Materials Standards - ASTM" - D 4541-93 (1995).

23. "British Standard Method of test for paints - BSI" - ISO 4624-1978 (1979).

24. "Book of American Society for Testing and Materials Standards - ASTM" - D 2834-72 (1975).

25. Blank, W.J. - J. Coat. Techn., 61, p. 119 (1994).

26. Satguru, G.; McMahon, J.; Padget, J.C. \& Coogan, R.G. - Amer. Paint \& Coat. J., mar, p. 47 (1993).

27. Weltzel, F.H. - "Introduction to Rubber-based Adhesives", in: Handbook of Adhesives, Irving Skeist (ed.), Reinhold Publishing Corporation, New York (1962).

Recebido: 06/07/99

Aprovado: 23/12/99 